\title{
NASA's Physics of the Cosmos and Cosmic Origins Technology Development Programs
}

\author{
Thai Pham*, Bernard Seery, and Opher Ganel, NASA Goddard Space Flight Center, \\ 8800 Greenbelt Road, Greenbelt, Maryland, U.S.A.
}

\begin{abstract}
The strategic astrophysics missions of the coming decades will help answer the questions "How did our universe begin and evolve?" and "How did galaxies, stars, and planets come to be?" Enabling these missions requires advances in key technologies far beyond the current state of the art. NASA's Physics of the Cosmos (PCOS) and Cosmic Origins (COR) Program Offices manage technology maturation projects funded through the Strategic Astrophysics Technology (SAT) program to accomplish such advances. The PCOS and COR Program Offices, residing at the NASA Goddard Space Flight Center (GSFC), were established in 2011, and serve as the implementation arm for the Astrophysics Division at NASA Headquarters. We present an overview of the Programs' technology development activities and the current technology investment portfolio of 23 technology advancements. We discuss the process for addressing communityprovided technology gaps and Technology Management Board (TMB)-vetted prioritization and investment recommendations that inform the SAT program. The process improves the transparency and relevance of our technology investments, provides the community a voice in the process, and promotes targeted external technology investments by defining needs and identifying customers. The Programs' priorities are driven by strategic direction from the Astrophysics Division, which is informed by the National Research Council's (NRC) "New Worlds, New Horizons in Astronomy and Astrophysics" (NWNH) 2010 Decadal Survey report [1], the Astrophysics Implementation Plan (AIP) [2] as updated, and the Astrophysics Roadmap "Enduring Quests, Daring Visions" [3]. These priorities include technology development for missions to study dark energy, gravitational waves, X-ray and inflation probe science, and large far-infrared (IR) and ultraviolet (UV)/optical/IR telescopes to conduct imaging and spectroscopy studies. The SAT program is the Astrophysics Division's main investment method to mature technologies that will be identified by study teams set up to inform the 2020 Decadal Survey process on several large astrophysics mission concepts.
\end{abstract}

Keywords: NASA, astronomy, astrophysics, technology, cosmic, origins, universe, stars, Hubble, galaxies, SAT

\section{INTRODUCTION}

Developing enabling technologies in advance of mission formulation is crucial to containing costs and maintaining schedules of large missions. As reported in a 2008 Space Review article [3], “...in the mid-1980s, NASA's budget office found that during the first 30 years of the civil space program, no project enjoyed less than a $40 \%$ cost overrun unless it was preceded by an investment in studies and technology of at least 5 to $10 \%$ of the actual project budget that eventually occurred." Having relatively mature key technologies in hand allows realistic planning and architecture design, which prevents major schedule delays and cost overruns.

NASA requires flight projects to achieve prior to Preliminary Design Review (PDR) Technology Readiness Level (TRL) of at least 6, defined as "System/sub-system model or prototype demonstration in a relevant environment" by systems engineering process NPR 7123.1B, Appendix E [4] for all their key technologies. Making this possible requires an adequately funded program that bridges the "mid-TRL gap" between TRL 3 (defined as "Analytical and experimental critical function and/or characteristic proof-of-concept" [4]) and TRL 5 (defined as "Component and/or breadboard validation in relevant environment." [4]). Unfortunately, the significant costs of the testing needed for TRL 5, combined with the lack of a flight project advocating for and willing to fund this work, make such technology maturation programs an easy target for cost-cutting efforts. Possibly as a result of such cost-cutting in the first decade of this century, noted in the NWNH, the Government Accountability Office (GAO) reported to Congress in March of 2012 on an assessment of 21 NASA projects with a combined life-cycle cost of more than $\$ 43$ billion, saying that a majority experienced significant schedule and cost overruns. The report stated, "Most of the projects that GAO reviewed did not meet technology maturity and design stability best practices criteria, which if followed can lessen cost and schedule risks

\footnotetext{
* bruce.t.pham@nasa.gov phone: +1-301-286-4809 fax: +1-301-286-1763
} 
faced by the project. Specifically, 10 of the 16 projects that held a preliminary design review moved forward without first maturing technologies. In addition, 13 of the 14 projects that held a critical design review did so without first achieving design stability" [5].

These observations illustrate the crucial need for well-funded programs dedicated for maturing key technologies up to TRL 5. Programs such as the SAT make it possible to pursue multiple parallel technological paths toward solving strategic technological gaps, mitigating the technical risks inherent in relying on any specific implementation concept. Setting up a flexible, responsive technology-development-management process mitigates the risk that science goals and performance requirements may change before a technology matures to the point of infusion into a funded flight project. As NWNH stressed, "Technology development is the engine powering advances in astronomy and astrophysics, from vastly extending the scientific reach of existing facilities to opening up new windows on the universe. All of the Astro2010 PPPs [Program Prioritization Panels] emphasized the critical importance of technology development, and each stressed the urgent need to augment existing funding levels to realize important programs essential to reducing the technical, cost, and schedule risk of planned missions. Mission- or project-specific technology development must reach an acceptable level before accurate costs can be determined, priorities set, and construction scheduled. Failure to develop adequately mature technology prior to a program start also leads to cost and schedule overruns" [1]. As noted in the 2015 Program Annual Technology Reports (PATRs) of both PCOS and COR, the work funded through SAT projects also trains the next generation of technologists and principal investigators for future Program strategic missions.

\section{NWNH AND PREPARING FOR THE 2020 DECADAL SURVEY}

Once per decade, a decadal survey of the astronomy and astrophysics community identifies the most compelling science goals, and recommends priorities for future missions, studies, and investments for the following decades. The 2010 report, NWNH, was informed by budgetary expectations of the time for NASA and the National Science Foundation (NSF). The AIP provided course corrections informed by budgetary developments unforeseen in 2010. The envisioned strategic missions would continue and build on the successes and breakthroughs of earlier major missions such as the Hubble Space Telescope (HST), Chandra, and Spitzer. The prioritized mission concepts would expand and extend our observational reach to new wavelengths, dramatically increased precision and stability, and even to a new signal type gravitational waves. Such ambitious projects require us to close the technology gaps between current state of the art and capabilities that are currently out of reach, such that the missions become feasible with acceptable budgets.

NWNH recommended optical and infrared survey telescopes for studying the nature of dark energy, making detailed observation of thousands of planetary systems, searching for exoplanets capable of sustaining life as we know it, and viewing the time-development of our universe as a "movie," rather than still images. A new window on the universe was recently opened by the ground-based Laser Interferometer Gravitational-Wave Observatory (LIGO), as it announced a breakthrough observation of gravitational waves emitted by two black holes, each on the order of 30 solar masses, as they spiraled into each other 1.3 billion light years from Earth. This confirmed general relativity predictions that motivate another NWNH priority, the Laser Interferometer Space Antenna (LISA), to be comprised of a trio of satellites in an equilateral triangle at least 1 million kilometers on a side. Using high-precision, high-stability laser measurements of the distance between them, LISA would measure the flexing of space as gravitational waves from super-massive black holes far outside the Milky Way galaxy traverse the formation. LISA would allow higher precision testing of general relativity and other possible theories of gravitation beyond what is achievable on the ground. Precision measurements at the short end of the electromagnetic spectrum, X-rays, and at its longer end, radio frequency, would allow studies of the earliest and farthest massive black holes, as well as subtle signatures of inflation left in the polarization of the cosmic microwave background (CMB). The NWNH large-scale and medium-scale space-mission priorities were as follows:

- (Large, currently managed by the Exoplanet Exploration Program, ExEP, but also related to PCOS) Wide-Field Infrared Survey Telescope (WFIRST), which it described as, "an observatory designed to settle essential questions in both exoplanet and dark energy research, and which will advance topics ranging from galaxy evolution to the study of objects within our own galaxy;"

- (Large) Augmenting NASA's Explorer Program, which it described as "a program that delivers a high level of scientific return on relatively moderate investment and that provides the capability to respond rapidly to new scientific and technical breakthroughs;"

- (Large, PCOS-related) LISA, described there as "a low-frequency gravitational wave observatory that will open an entirely new window on the cosmos by measuring ripples in space-time caused by many new sources, including nearby white dwarf stars, and will probe the nature of black holes;" 
- (Large, PCOS-related) International X-ray Observatory (IXO), "a powerful X-ray telescope that will transform our understanding of hot gas associated with stars and galaxies in all evolutionary stages;"

- (Medium, ExEP-related) New Worlds Technology Development Program, "a competed program to lay the technical and scientific foundation for a future mission to study nearby Earth-like planets;" and

- (Medium, PCOS-related) Inflation Probe Technology Development Program, "a competed program designed to prepare for a potential next-decade cosmic microwave background mission to study the epoch of inflation."

In January, NASA Astrophysics Division Director Paul Hertz announced that NASA is initiating studies of four large mission concepts, kicking off Science and Technology Definition Teams (STDTs) to inform the 2020 decadal survey:

- Far-IR Surveyor (study being carried out at GSFC);

- Habitable Exoplanet Imaging Mission (study being carried out at JPL);

- Large UV/Optical/IR (LUVOIR) Surveyor (study being carried out at GSFC); and

- X-ray Surveyor (study being carried out at MSFC).

The STDTs were charged to develop science cases, flow those into mission requirements, vet lists of technology needs, and identify science vs. cost/capability trades. Technology-development-related study deliverables include the following:

- (Optional) "Deliver Initial Technology Gap Assessment" - June 30, 2016;

- (Required) "Complete Concept Maturity Level 2 Audit... Identify, quantify and prioritize technology gaps for 2017 technology cycle" - February 2017;

- (Optional) "Update Technology Gap Assessments" - June 2017;

- (Required) "Interim report...Deliver initial technology roadmaps" - early December 2017;

- (Required) "Update Gap Assessments... In support of 2018 technology cycle" - June 2018; and

- (Required) "Final Report... Finalize technology roadmaps, tech plan and cost estimates for technology maturity" - January 2019.

The STDTs are not intended to compete against each other, but rather provide the 2020 decadal survey panel with four compelling and executable concepts so that science can be adequately prioritized.

\section{PHYSICS OF THE COSMOS AND COSMIC ORIGINS PROGRAMS}

NASA's Astrophysics Division addresses three fundamental and enduring questions:

1. How does our universe work?

2. How did we get here?

3. Are we alone?

To address these questions, the Astrophysics Division established three programs, each with its own portfolio, missions, and technology development activities managed by a dedicated Program Office. These are, respectively, the PCOS, COR, and ExEP offices. In this paper, we discuss the technology development process and activities of the PCOS and COR Program Offices, funded through Technology Development for PCOS (TPCOS) and Technology Development for COR (TCOR). ExEP technology development projects are funded via Technology Development for Exoplanet Missions (TDEM).

The PCOS and COR Program Offices strive for transparency; informing all stakeholders of their processes, evolving technology needs and priorities, technology investments, and progress in funded projects. These are all reported by Program websites as well as PATRs ${ }^{\dagger}$. The Program Offices follow the strategic guidance of the decadal survey as directed by the NASA HQ's Astrophysics Division. This ensures prioritization and funding of the most relevant key technologies, enabling and enhancing the strategic astrophysics missions of the coming decades. The process encourages non-NASA technology developers, including industry, to invest in high-priority Astrophysics technologies by identifying existing and emerging needs, and by identifying potential customers and possible technology providers.

\footnotetext{
${ }^{+}$Available through pcos.gsfc.nasa.gov/technology and cor.gsfc.nasa.gov/docs/
} 


\subsection{The PCOS Program}

PCOS science includes cosmology, high-energy astrophysics, physics of massive objects such as black holes and neutron stars, dark matter, dark energy, and gravitational waves. This science theme uses the cosmic scale and extreme objects and environments as a laboratory to study the basic properties of nature. The program focuses on activities, technologies, and projects intended to enhance our understanding of how the universe works by probing Einstein's General Theory of Relativity, the nature of space-time, the behavior of matter and energy in extreme environments, dark energy and the accelerating universe, precision measurements of cosmological parameters governing the evolution of the universe, tests of the inflation hypothesis, and the connection between galaxies and supermassive black holes.

The PCOS Program encompasses multiple scientific missions, below, aimed at meeting Program objectives, each with unique scientific capabilities and goals. The Program was established to integrate those missions into a cohesive effort enabling each project to build on the technological and scientific legacy of its contemporaries and predecessors. Each project operates independently toward its unique mission objectives, which contribute to overall Program objectives.

- Chandra, X-ray, launched in 1999

- X-ray Multi-mirror Mission-Newton, X-ray, launched in 1999

- Fermi Gamma-Ray Space Telescope, gamma ray, launched in 2008

- Space Technology 7/ LISA Pathfinder (ST7/LPF), gravitational waves, ESA mission with US participation, launched in 2015

- Euclid, visible and Near-IR, ESA mission with US participation, planned to launch in 2020

- WFIRST, Near-IR, managed by the ExEP PO and addresses PCOS science, planned to launch in mid-2020s

- Athena, X-ray, ESA mission with US participation, planned to launch in 2028

As detailed above, NWNH suggested high priority be given to several elements addressing PCOS science - LISA (gravitational waves, most likely to be addressed by ESA's L3 mission foreseen to be launched in mid-2030s), IXO (Xray, expected to be addressed by ESA's Athena mission (see above), and possibly by X-ray Surveyor in the 2030s or later), and Inflation Probe technology development (microwave, no current mission timeline). Of the four STDTs mentioned above, the X-ray Surveyor fits within the PCOS purview.

The PCOS Program Office solicits ongoing community input and engagement through the PCOS Program Analysis Group (PhysPAG), constituted by the NASA Astrophysics Subcommittee, the PCOS website (including a downloadable technology gaps form ${ }^{\ddagger}$, and PATRs.

\subsection{The COR Program}

Encompassing most of the field of astronomy, COR science seeks to understand the origin and evolution of the universe from the Big Bang to how it appears today; to determine how the universe expanded from a singularity into a vast cosmic web of dark matter enmeshed with galaxies and intergalactic material, forming, merging, and evolving over time. At smaller scales, COR studies how clouds of dust coalesce and collapse into stars and planets, how the heavier elements crucial for life are synthesized in the cores of stars, and how the death of stars flings them out to where they provide the building blocks essential for life.

The program manages and integrates space, airborne, and ground-based missions and activities, synergistically addressing the above science questions. Current and planned missions and platforms addressing COR science include:

- Hubble Space Telescope (HST), visible light, launched in 1990

- Spitzer Space Telescope, IR, launched in 2003, cryogen exhausted in 2009 but shortest wavelength observations continue as the "Spitzer Warm Mission"

- Herschel Space Observatory, far-IR and sub-millimeter, ESA mission with US participation, launched in 2009, completed operations in 2013, but data analysis continues (planned to conclude in 2017)

- Stratospheric Observatory for Infrared Astronomy (SOFIA), IR, world's largest airborne observatory, saw first light in 2010, 20-year planned lifetime

- James Webb Space Telescope (JWST), IR, planned to launch in 2018

* The Program solicits technology gap inputs by June 1 for each year's prioritization cycle, but accepts input year-round (download form at http://pcos.gsfc.nasa.gov/technology/PCOS_Tech_Gap_Form_-_your_last_name_new.docx). 
NWNH recommendations within or overlapping COR science interests include Cosmic Dawn, WFIRST, a 4m-class UV/Vis space telescope, JAXA's SPICA mission, and augmentation of the Explorer program. Both the Far-IR Surveyor and the LUVOIR Surveyor would address COR science topics. COR technology developments being developed through SAT projects, past and present, include detectors measuring IR, sub-millimeter, and UV wavelengths, multi-object UV spectrometers, and advanced UV-reflective coatings. The COR program also solicits community input, and engages the community in ongoing discussions through the COR Program Analysis Group (COPAG), the COR website (including a downloadable technology gaps form ${ }^{\S}$ ), and PATRs.

\section{PCOS AND COR TECHNOLOGY MANAGEMENT PROCESS}

PCOS and COR have developed and continue to improve an annual process for identifying technology gaps, prioritizing them, and managing technology maturation projects (Fig. 1). The Programs engage their communities through PhysPAG and COPAG workshops and other public meetings, and solicit throughout the year community input on technology gaps. The programs' TMBs prioritize the technology gaps based on a set of four criteria (below). This prioritization is carried out annually, in order to maintain relevance in the face of constantly evolving science goals, technology developments, and budgetary realities. STDT technology gap submissions will be assessed and prioritized side-by-side with gaps submitted by the community at large, which will require some slight modifications in the details of the prioritization process. Finally, the Program Offices encourage and promote infusion of newly matured technologies into flight missions as appropriate.

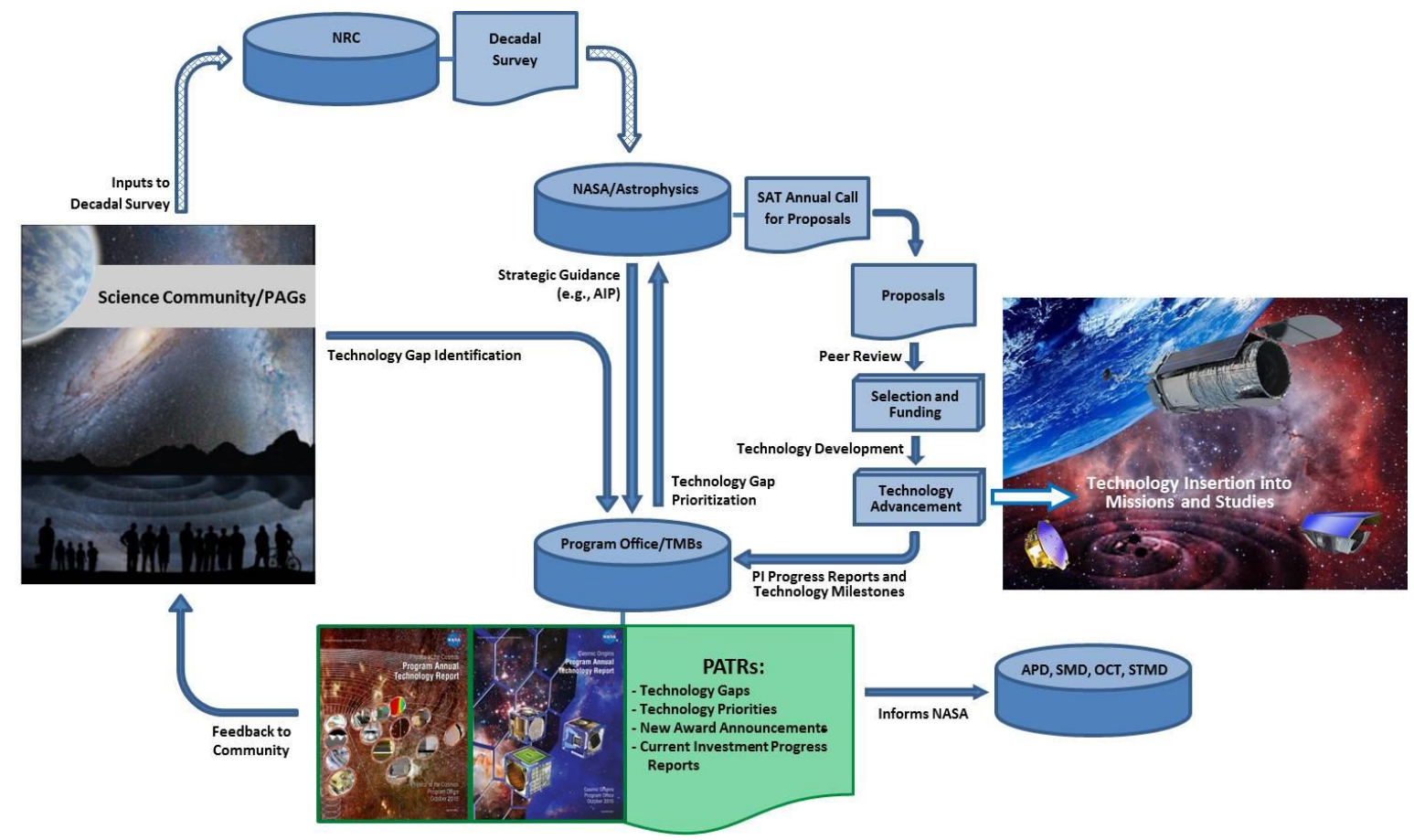

Figure 1. The PCOS and COR technology management process promotes maturation of the highest priority technologies until they can be infused into flight projects, giving a central role to the community.

The TMBs consist of senior staff from NASA HQ Astrophysics Division and the Program Offices, along with subject matter experts, program scientists, technologists, and systems engineers. The TMBs carry out multiple advisory tasks:

- Technology gap prioritization;

- Program-level oversight;

- Review of technology development and vetting PI assessment of TRL advances; and

- Input on technology issues and recommendations to the Program Offices and the Astrophysics Division.

$\S$ The Program solicits technology gap inputs by June 1 for each year's prioritization cycle, but accepts input year-round (download form at http://cor.gsfc.nasa.gov/technology/COR_Tech_Gap_Form_-_your_last_name.docx). 
The criteria used by the TMB are described in the PATR as follows (with weight factors in parentheses).

- Strategic Alignment (10): How well does the technology align with PCOS science and/or programmatic priorities of the AIP or current programmatic assessment (e.g., the Astrophysics Roadmap)?

- Benefits and Impacts (8): How much impact does the technology have on applicable missions? To what degree does it enable and/or enhance achievable science objectives, reduce cost, and/or reduce mission risks?

- Scope of Applicability (3): How crosscutting is the technology? How many Astrophysics programs and/or mission concepts would it benefit?

- Urgency (4): When are the enabled/enhanced missions' launches anticipated and/or by when do other schedule drivers require progress?

These criteria and the specifics of their application are reviewed annually and adjusted as needed, in response to new developments and Astrophysics Division guidance.

\section{TECHNOLOGY GAPS AS OF 2015}

The 2015 PCOS and COR PATRs rank by priority 22 and 20 technology gaps, respectively, with each list divided into three priority tiers. The following are the top tier technology gaps in the two programs (see 2015 PATRs for complete lists including tiers 2 and 3 ).

PCOS Priority 1: Technology gaps determined to be of highest interest to the PCOS Program.

1. High-power, narrow-line-width laser sources

2. Highly stable, low-stray-light telescope

3. Large-format, high-spectral-resolution, small-pixel X-ray focal-plane arrays

4. Affordable, lightweight, high-resolution X-ray optics

5. Advanced millimeter-wave focal plane arrays for CMB polarimetry

6. High-efficiency cooling systems covering the range $20 \mathrm{~K}$ to under $1 \mathrm{~K}$

COR Priority 1: Technology gaps determined to be of highest interest to the COR Program.

1. Large-format, low-noise and ultralow-noise far-IR direct detectors

2. Band-shaping and dichroic filters for UV/Vis

3. Heterodyne far-IR detector arrays and related technologies

4. High-QE, rad-hard, large-format, non-photon-counting UVOIR detectors

5. Photon-counting large-format UV detectors

6. High-efficiency UV multi-object spectrometers

7. High-reflectivity mirror coatings for UV/Vis/near-IR

Priority 2 gaps, if closed, would have a somewhat lesser impact on strategic missions, or may enable missions further in the future. However, they are still worthy of pursuit subject to funding availability ( 7 technologies for PCOS, 8 for COR). Historically, most technologies solicited in the Research Opportunities in Space and Earth Sciences (ROSES) SAT announcement of opportunity were taken from the top priority tier, but many came from priority tier 2. Priority 3 technologies may be supportive of program objectives but for various reasons do not warrant investment unless significant additional funding becomes available ( 9 for PCOS, 5 for COR). SAT proposals are assessed on 1) overall scientific and technical merit; 2) programmatic relevance; and 3) reasonableness of proposed cost.

The Program Offices encourage gap submitters to include as much of the requested information as possible, as this improves prioritization accuracy, usually in favor of more complete entries. Entries should be of missing capabilities, not specific implementations. Complete information that includes clear, specific, measurable requirements increases the likelihood that NASA HQ will solicit the most critical technologies and proposers will be better able to assess candidate technologies for submission. This, in turn, will lead to more and better technology infusion into strategic missions. Where specifying exact technical parameters is problematic, ranges or targets for relevant parameters would be useful. Submissions should detail current state-of-the-art capability and state how the stated need exceeds it. Providing a list of strategic astrophysics missions enabled or enhanced by the technology also improves the probability of higher priority.

Tables 1 and 2 show the currently funded programs for PCOS and COR, respectively. 


\begin{tabular}{|c|c|c|c|c|c|c|}
\hline $\begin{array}{l}\text { Funding } \\
\text { Source }\end{array}$ & Technology Development Title & $\begin{array}{l}\text { Principal } \\
\text { Investigator }\end{array}$ & Org & $\begin{array}{l}\text { Start Year, } \\
\text { Duration }\end{array}$ & Science Area & Tech Area \\
\hline SAT2010 & $\begin{array}{l}\text { Directly-Deposited Blocking Filters for Imaging X-ray Detectors: } \\
\text { Technology Development for the International X-ray Observatory }\end{array}$ & Mark Bautz & MIT & $\begin{array}{l}\text { FY2012, } 4 \\
\text { years }\end{array}$ & X-ray & Detector \\
\hline APRA2011 & $\begin{array}{l}\text { Adjustable Grazing Incidence X-ray Optics with } 0.5 \text { Arc Second } \\
\text { Resolution }\end{array}$ & Paul Reid & SAO & $\begin{array}{l}\text { FY2013, } 3 \\
\text { years }\end{array}$ & X-ray & Optics \\
\hline SAT2012 & $\begin{array}{l}\text { Phase Measurement System Development for Interferometric } \\
\text { Gravitational Wave Detectors }\end{array}$ & William Klipstein & JPL & $\begin{array}{l}\text { FY2014, } 3 \\
\text { years }\end{array}$ & GW & Electronics \\
\hline SAT2012 & Demonstration of a TRL 5 Laser System for eLISA & Jordan Camp & GSFC & $\begin{array}{l}\text { FY2014, } 2 \\
\text { years }\end{array}$ & GW & Laser \\
\hline $\begin{array}{l}\text { SAT2012 } \\
\text { SAT2010 }\end{array}$ & $\begin{array}{l}\text { Advanced Antenna-Coupled Superconducting Detector Arrays for CMB } \\
\text { Polarimetry }\end{array}$ & Jamie Bock & JPL & $\begin{array}{l}\mathrm{FY} 2014,2 \\
\text { years }\end{array}$ & CMB & Detectors \\
\hline $\begin{array}{l}\text { SAT2013 } \\
\text { SAT2010 }\end{array}$ & Reflection Grating Modules: Alignment and Testing & Randy McEntaffer & U. of lowa & $\begin{array}{l}\text { FY2015, } 2 \\
\text { years }\end{array}$ & X-ray & Optics \\
\hline $\begin{array}{l}\text { SAT2013 } \\
\text { SAT2010 }\end{array}$ & Advanced Packaging for Critical Angle X-ray Transmission Gratings & Mark Schattenburg & MIT & $\begin{array}{l}\text { FY2015, } 2 \\
\text { years }\end{array}$ & X-ray & Optics \\
\hline SAT2013 & $\begin{array}{l}\text { Technology Development for an AC-Multiplexed Calorimeter for } \\
\text { ATHENA }\end{array}$ & Joel Ullom & NIST & $\begin{array}{l}\text { FY2015, } 2 \\
\text { years }\end{array}$ & X-ray & Detector \\
\hline $\begin{array}{c}\text { SAT2013 } \\
\text { APRA2011 }\end{array}$ & $\begin{array}{l}\text { Development of } 0.5 \text { Arc-second Adjustable Grazing Incidence X-ray } \\
\text { Mirrors for the SMART-X Mission Concept }\end{array}$ & Paul Reid & SAO & $\begin{array}{l}\text { FY2015, } 3 \\
\text { years }\end{array}$ & X-ray & Optics \\
\hline $\begin{array}{l}\text { SAT2013 } \\
\text { SAT2011 }\end{array}$ & Affordable and Lightweight High-Resolution Astronomical X-Ray Optics & William Zhang & GSFC & $\begin{array}{l}\text { FY2015, } 2 \\
\text { years }\end{array}$ & X-ray & Optics \\
\hline SAT2013 & Fast Event Recognition for the ATHENA Wide Field Imager & David Burrows & PSU & $\begin{array}{l}\text { FY2015, } 2 \\
\text { years }\end{array}$ & X-ray & Detector \\
\hline $\begin{array}{l}\text { SAT2014 } \\
\text { SAT2012 } \\
\text { SAT2010 }\end{array}$ & $\begin{array}{l}\text { Superconducting Antenna-Coupled Detectors and Readouts for Space- } \\
\text { Borne CMB Polarimetry }\end{array}$ & James Bock & JPL & $\begin{array}{l}\text { FY2016, } 2 \\
\text { years }\end{array}$ & $\mathrm{CMB}$ & Detectors \\
\hline $\begin{array}{l}\text { SAT2014 } \\
\text { SAT2011 }\end{array}$ & $\begin{array}{l}\text { Telescope Dimensional Stability Study for a Space-based Gravitational } \\
\text { Wave Mission }\end{array}$ & Jeffrey Livas & GSFC & $\begin{array}{l}\text { FY2016, } 2 \\
\text { years }\end{array}$ & GW & Telescope \\
\hline SAT2014 & $\begin{array}{l}\text { High Efficiency Feedhorn-Coupled TES-based Detectors for CMB } \\
\text { Polarization Measurements }\end{array}$ & Edward Wollack & GSFC & $\begin{array}{l}\text { FY2016, } 2 \\
\text { years }\end{array}$ & CMB & Detector \\
\hline $\begin{array}{l}\text { Targeted } \\
2016\end{array}$ & $\begin{array}{l}\text { Providing Enabling and Enhancing Technologies for a Demonstration } \\
\text { Model of the Athena X-IFU }\end{array}$ & Caroline Kilbourne & GSFC & $\begin{array}{l}\text { FY2016, } 2 \\
\text { years }\end{array}$ & X-ray & Detector \\
\hline
\end{tabular}

Table 1. Currently funded PCOS technology projects.

\begin{tabular}{|c|c|c|c|c|c|c|}
\hline $\begin{array}{l}\text { Funding } \\
\text { Source }\end{array}$ & Technology Development Title & $\begin{array}{l}\text { Principal } \\
\text { Investigator }\end{array}$ & Org & $\begin{array}{l}\text { Start Year, } \\
\text { Duration }\end{array}$ & $\begin{array}{l}\text { Science } \\
\text { Area }\end{array}$ & $\begin{array}{l}\text { Tech } \\
\text { Area }\end{array}$ \\
\hline SAT2010 & $\begin{array}{l}\text { High performance cross-strip micro-channel plate detector systems } \\
\text { for spaceflight experiments. }\end{array}$ & John Vallerga & UCB & $\begin{array}{l}\text { FY2012, } 4 \\
\text { years }\end{array}$ & UV & Detector \\
\hline SAT2011 & $\begin{array}{l}\text { Ultraviolet coatings, materials and processes for advanced } \\
\text { telescope optics }\end{array}$ & $\begin{array}{l}\text { Kunjithapatham } \\
\text { Balasubramanian }\end{array}$ & JPL & $\begin{array}{l}\text { FY2013, } 3 \\
\text { years }\end{array}$ & UV & $\begin{array}{l}\text { Optical } \\
\text { Coating }\end{array}$ \\
\hline SAT2011 & $\begin{array}{l}\text { Kinetic Inductance Detector Imaging Arrays for Far-Infrared } \\
\text { Astrophysics }\end{array}$ & $\begin{array}{l}\text { Jonas } \\
\text { Zmuidzinas }\end{array}$ & JPL & $\begin{array}{l}\text { FY2013, } 3 \\
\text { years }\end{array}$ & Far-IR & Detector \\
\hline SAT2012 & A Far-Infrared Heterodyne Array Receiver for CII and OI Mapping & Imran Mehdi & JPL & $\begin{array}{l}\text { FY2014, } 3 \\
\text { years }\end{array}$ & Far-IR & $\begin{array}{l}\text { Detector } \\
\mathrm{s}\end{array}$ \\
\hline SAT2012 & $\begin{array}{l}\text { Deployment of Digital Micromirror Device (DMD) Arrays For Use In } \\
\text { Future Space Missions }\end{array}$ & Zoran Ninkov & RIT & $\begin{array}{l}\text { FY2014, } 2 \\
\text { years }\end{array}$ & UV & Detector \\
\hline $\begin{array}{l}\text { SAT2012 } \\
\text { SAT2010 }\end{array}$ & Advanced Mirror Technology Development Phase 2 & Phil Stahl & MSFC & $\begin{array}{l}\text { FY2014, } 3 \\
\text { years }\end{array}$ & LUVOIR & Optics \\
\hline SAT2014 & Raising the Technology Readiness Level of $4.7-\mathrm{THz}$ local oscillators & Qing $\mathrm{Hu}$ & MIT & $\begin{array}{l}\text { FY2016, } 3 \\
\text { years }\end{array}$ & Far-IR & Detector \\
\hline $\begin{array}{l}\text { SAT2014 } \\
\text { SAT2010 }\end{array}$ & $\begin{array}{l}\text { Development of Large Area }(100 \times 100 \mathrm{~mm}) \text { photon counting UV } \\
\text { detectors }\end{array}$ & John Vallerga & UCB & $\begin{array}{l}\text { FY2016, } 2 \\
\text { years }\end{array}$ & UV & Detector \\
\hline SAT2014 & $\begin{array}{l}\text { Building a Better ALD - use of Plasma Enhanced ALD to Construct } \\
\text { Efficient Interference Filters for the FUV }\end{array}$ & Paul Scowen & ASU & $\begin{array}{l}\text { FY2016, } 3 \\
\text { years }\end{array}$ & UV & $\begin{array}{l}\text { Optical } \\
\text { Coating }\end{array}$ \\
\hline $\begin{array}{l}\text { SAT2014 } \\
\text { SAT2011 }\end{array}$ & $\begin{array}{l}\text { Advanced FUVUV/Visible Photon Counting and Ultralow Noise } \\
\text { Detectors }\end{array}$ & Shouleh Nikzad & JPL & $\begin{array}{l}\text { FY2016, } 3 \\
\text { years }\end{array}$ & LUVOIR & Detector \\
\hline SAT2014 & Ultra-Stable Structure & Babak Saif & GSFC & $\begin{array}{l}\text { FY2016, } 4 \\
\text { years }\end{array}$ & LUVOIR & $\begin{array}{l}\text { Stable } \\
\text { Structure }\end{array}$ \\
\hline
\end{tabular}

Table 2. Currently funded COR technology projects. 


\section{SAT SOLICITATIONS, FUNDING AND BENEFITS}

The SAT Program solicits technology-maturation proposals through ROSES. Up to the 2014 SAT cycle, 65 COR and 71 PCOS SAT proposals were received, with 15 COR and 22 PCOS projects selected. In the 2015 SAT cycle, 12 COR proposals and 10 PCOS proposals were received, with selections not yet announced as of this writing. Beyond maturing strategic technologies, most SAT principal investigators report their projects let them leverage funding from internal and external (NASA and non-NASA) sources; hire students and post-docs, training the future astrophysics workforce; and create research collaborations and industry partnerships in support of Program goals.

\section{OUTLOOK FOR THE FUTURE}

Bridging the gap between "blue-sky" concept (TRL 1-3) and implementable (TRL 6-9) technologies in key areas enables drafting plausible schedules and budgets for strategic missions, and decreases the risk of funded missions significantly exceeding their schedules and budgets. Successful maturation of the above-listed technologies will enable and enhance strategic missions planned to measure electromagnetic and gravitational-wave signals from cosmic sources. The STDTs studying four large mission concepts will provide compelling science and programmatic cases for some of the most exciting paths forward, and identify any other technologies needed for these missions to succeed. Whichever of the four concepts (or different one) is prioritized by the 2020 decadal survey report, it can be expected to enable breakthroughs in our understanding of the origin of the universe and the physics laws that govern it.

\section{ACKNOWLEDGEMENTS}

The NASA Headquarters Astrophysics Division funds all activities described in this paper, including the SAT program and the Program Offices located at GSFC. We are grateful to all the PIs and their teams for their many compelling proposals, and for adhering to the technology management process described above. Their willingness to support and improve our process has been and continues to be essential to the success of the programs.

\section{REFERENCES}

[1] National Research Council, "New Worlds, New Horizons in Astronomy and Astrophysics," Washington, DC: The National Academies Press, 2010. Available at www.nap.edu/catalog.php?record_id=12951. Accessed March 2016.

[2] Astrophysics Division, Science Mission Directorate, NASA Headquarters, "Astrophysics Implementation Plan," as corrected April 15, 2013. Available at science.nasa.gov/media/medialibrary/2013/04/15/secureImpPlan_R2_15Apr2013.pdf. Accessed March 2016.

[3] E. Agol, N. Batalha, J. Bean, M. Bentz, N. Cornish, A. Dressler, E. Figueroa-Feliciano, S. Gaudi, O. Guyon, D. Hartmann, J. Kalirai, M. Niemack, F. Ozel, C. Reynolds, A. Roberge, K. Sheth. A. Straughn, D. Weinberg, and J. Zmuidzinas, "Enduring Quests-Daring Visions (NASA Astrophysics in the Next Three Decades)," arXiv:1401.3741v1 [astro-ph.IM]. Accessed December 2015.

[4] John C. Mankins, "The critical role of advanced technology investments in preventing spaceflight program cost overrun,” The Space Review, December 1, 2008. Available at www.thespacereview.com/article/1262/1. Accessed May 2014.

[5] NODIS library; nodis3.gsfc.nasa.gov/displayDir.cfm?t=NPR\&c=7123\&s=1B

[6] United States Government Accountability Office, "NASA Assessments of Selected Large-Scale Projects, GAO-12207SP", March 1, 2012. Available at www.gao.gov/assets/590/589016.pdf. Accessed March 2016. 